\title{
A Robust Method for S1/S2 Heart Sounds Detection Without ECG Reference Based on Music Beat Tracking
}

\author{
Constantin Barabaşa \\ Faculty of Electronics Telecommunications and Information \\ technology \\ Gh. Asachi Technical University of Iaşi \\ Iaşi, Romania \\ cbarabasa@etti.tuiasi ro
}

\author{
Maria Jafari, Mark D. Plumbley \\ Centre for Digital Music \\ Queen Mary University of London \\ London, United Kingdom \\ maria.jafari@eecs.qmul.ac.uk, \\ mark.plumbley@eecs.qmul.ac.uk
}

\begin{abstract}
We present a robust method for the detection of the first and second heart sounds (s1 and s2), without ECG reference, based on a music beat tracking algorithm. An intermediate representation of the input signal is first calculated by using an onset detection function based on complex spectral difference. A music beat tracking algorithm is then used to determine the location of the first heart sound. The beat tracker works in two steps, it first calculates the beat period and then finds the temporal beat alignment. Once the first sound is detected, inverse Gaussian weights are applied to the onset function on the detected positions and the algorithm is run again to find the second heart sound. At the last step s1 and s2 labels are attributed to the detected sounds. The algorithm was evaluated in terms of location accuracy as well as sensitivity and specificity and the results showed good results even in the presence of murmurs or noisy signals.
\end{abstract}

Keywords-heart sound; detection; beat tracking; onset detection function

\section{INTRODUCTION}

Heart sound auscultation, i.e. the use of a stethoscope by a trained physician for listening to the sound the heart produces, is one of the most reliable and successful means of early diagnosis of heart dysfunctions. There have been increasing efforts towards finding successful automated heart sound classification algorithms and a key contributing factor to that success is robust and reliable detection of the elements that make up a heart sound. These elements are the first heart sound (s1), the systolic period (sp), the second heart sound (s2) and the diastolic period (dp), in this time sequence (Figure 1). The s1 and s2 are often described as the $l u b$ and $d u b$ that make up the $l u b-d u b$ heart sound most people are familiar with, and the systolic and diastolic periods are the pauses between them.

There are two distinct approaches to $\mathrm{s} 1 / \mathrm{s} 2$ detection depending on whether or not the ECG is used as a reference (i.e. key ECG features that occur at the same time as features in heart sounds are detected instead).

The first one relies on the temporal correspondence between ECG features and the s1 and s2 [8]. The heart sound and ECG must be recorded simultaneously and because ECG analysis methods are well established, finding the location of ECG features is straightforward. The second approach, referred as independent segmentation $[4,5,6,7]$, identifies the s1 and s2 heart sounds without any supplementary information or signals except from the heart sound itself.

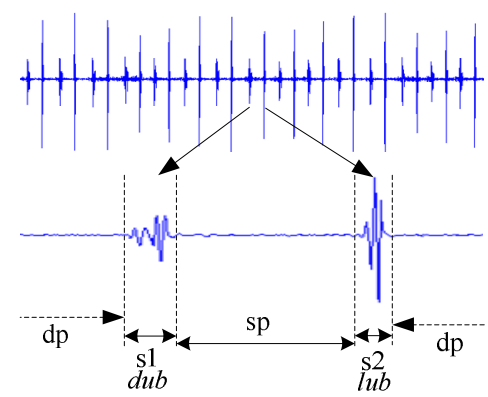

Figure 1. A normal heart sound and a zoomed in view at a beat cycle

The work presented here tackles the second situation. There are several advantages to using independent segmentation, the most important being that patient comfort is not compromised, since only an electronic stethoscope is needed, and it eliminates the need for additional hardware like an ECG machine.

Several methods can be found in the literature where independent segmentation techniques are applied, based either on heart sound envelogram (envelope calculated using the normalized average Shannon energy) [3], or on frequency domain analysis $[4,5,6,7]$. These methods perform well (92 to $94 \%$ correct detections), however they either have been developed for a particular situation (only on non pathological situations, or a specific pathology), or suffer from a degradation in performance in the presence of heart murmurs (pathological noises produced by turbulent flow of blood), or noisy environments.

Music analysis is an area where there is much interest in research and looking at the main characteristics of the heart sound, namely the periodicity and shape, has lead us to the idea that the concept of beat tracking used in music analysis can be successfully modified and applied to heart sound segmentation. In music, beat tracking is the computational equivalent of tapping one's foot in time to music, and heart sounds present themselves like an ideal candidate since they are repetitive in nature and far less complex than music.

\section{METHOD}

We propose a robust method, which does not suffer from performance degradation regardless of the situation at least 
from the specificity and selectivity point of view, for heart sound segmentation and s1/s2 detection, without ECG reference, based on a music beat tracking algorithm introduced by Davies and Plumbley [1]. Our method first calculates an intermediate representation of the input signal by using an onset detection function based on complex spectral difference. The beat tracking algorithm is then used to determine the location of one of the two heart sounds. Once the first sound is detected, inverse Gaussian weights are applied to the onset function on the detected positions and the algorithm is run again to find the second heart sound. Because the order in which the s1 and s2 heart sounds are detected is not predictable, at the last step s1 and s2 labels must be correctly attributed to the detected sounds. This decision is made by analyzing the length of the intervals between the two sounds. We shall use the notations $s^{\prime}$ and $s^{\prime \prime}$ to denote the two heart sounds, relating to the order in which they are detected.

Within the first stage of the detection algorithm, the input signal is converted into a more useful and compact representation than the time domain. Audio beat tracking algorithms refer to this representation as an onset detection function [3]. We have chosen the complex spectral difference as being the most suitable for our purpose because by working in the complex domain, detection can be made either as a result of energy change in the magnitude spectrum or a modification in phase values in the phase spectrum.

In order to calculate the onset detection function, a moving window of size $\mathrm{N}$ and step $\mathrm{N} / 8$ is used, creating a $12.5 \%$ overlap at each step. In order to minimize frequency leakage a Hamming window has been chosen. At step k, the onset detection function $\Gamma(k)$ is calculated as the sum of the Euclidean distance between the current frame spectrum $S_{m}(k)$ and previous frame spectrum $S_{m}(k-1)$ for all frequency bins $\mathrm{m}$

$$
\Gamma(k)=\sum_{m=1}^{M}\left|S_{m}(k)-S_{m}(k-1)\right|^{2}
$$

Also, to allow operation regardless of sampling frequency the onset detection function is calculated in such a way that a fixed time resolution per detection function sample is obtained. Several window sizes have been tested, and an optimum result has been obtained for values of

$$
N \cong F_{S} / 12
$$

samples. Larger window values tend to favor false negative detections, while smaller values lead to false positives. An example of heart sound and corresponding onset detection function is shown in Figure 2 (a) and (b).

The beat tracking algorithm used here works in two steps: it first extracts the beat period and then finds the correct beat alignment. The onset detection function $D F$ is divided into overlapping analysis frames with a step equal to half the frame size. For each frame, an autocorrelation function is calculated and filtered through a comb filterbank. Then to achieve well defined peaks and discard the least significant ones, it is passed through an adaptive moving mean threshold and half wave rectified. (a)

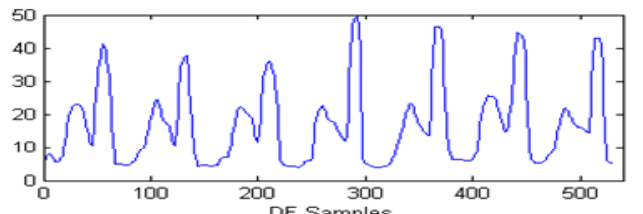

(b)

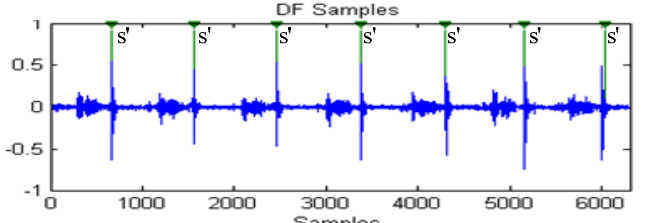

(c)

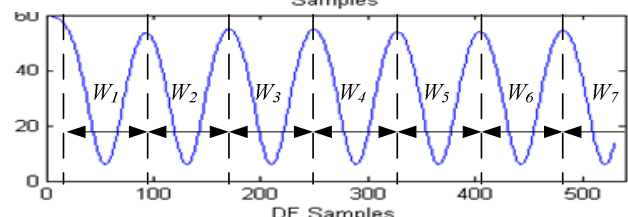

(d)
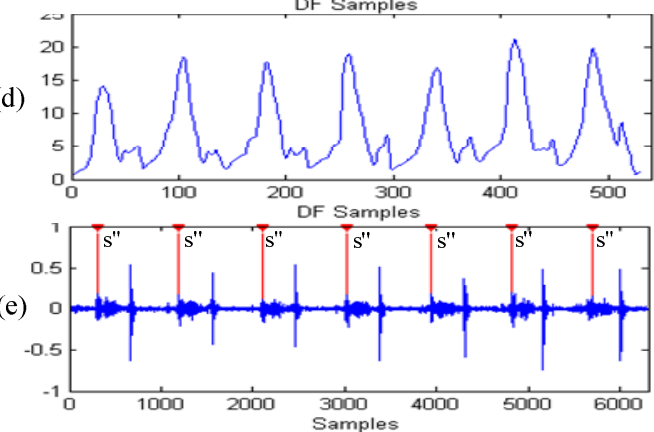

Figure 2. (a) DF onset detection function; (b) Heart sound recording and first detected heart sound $-s^{\prime}$; (c) $W_{i}$ applied for all $s^{\prime}$ positions; (d) DF' onset detection function; (g) Heart sound recording and second detected heart sound $-s^{\prime \prime}$

The beat period is identified as the index of the maximum value. After the beat period is calculated for all the recording, the locations of the first heart sound are determined using dynamic programming and the same comb filtering technique mentioned above. We will not go into further details as the beat tracking method is described thoroughly in [1].

Since s1 and s2 are the main features in the heart sound that generate onset, by applying the beat tracking algorithm, we expect that the detected beat position will correspond to one or the other of the two. Once one of the two heart sounds, $s^{\prime}$ (figure 2b), is detected throughout the recording, inverse Gaussian weights are applied to the onset detection function (figure 2c), centered on each detected location. A weighting vector $W_{i}$ of length $\mathrm{D}$, is constructed for every detected $s^{\prime}$ according to

$$
W_{i}(x)=1-\delta e^{-\frac{\left(x-\mu_{i}\right)^{2}}{2 \sigma^{2}}}, x=1 . . D
$$

where $\mu_{i}$, the median, is the position of the $\mathrm{i}^{\text {th }}$ detected $s^{\prime}$ in $D F$ samples, $\sigma$ is the variance of the distribution, and was found to yield the best results for values around $T / 5$, where $T$ is the average detected beat period in $D F$ samples. $\delta$ is a scaling factor and $\mathrm{D}$ is the length of the onset detection function. A value of 0.95 has been used for $\delta$, however this can vary and it represents how much $D F$ is attenuated at the current beat location. Larger values tend not to have any 
influence on the detection but smaller values affect performance. The weighted detection function $D F^{\prime}$ will be obtained by successively applying $W_{i}$ to $D F$ (figure $2 \mathrm{~d}$ ). After calculating $D F^{\prime}$, the beat tracking algorithm is ran one more time, this time using $D F^{\prime}$ instead of $D F$ and the locations of the second heart sound, $s^{\prime \prime}$ are determined (figure 2e). The order in which $\mathrm{s} 1$ and $\mathrm{s} 2$ are detected cannot be predicted because the beat tracker will detect either of them with equal probability on the first run, so correct labels must be assigned to $s^{\prime}$ and $s^{\prime \prime}$. For that purpose, the average distance between an $s^{\prime}$ and the following and preceding $s^{\prime \prime}$ will be used. If we consider $\tau_{1}^{i}$ as the distance in samples between $s_{i}^{\prime}$ and the preceding $s^{\prime \prime}, \tau_{2}^{i}$ as the distance between $s^{\prime}{ }_{i}$ and the following $s^{\prime \prime}$, and $i=1$.. I with $I$ being the total number of detected $s^{\prime}$ except for the first and last, then the sign of $\tilde{\tau}$ defined as

$$
\tilde{\tau}=\frac{1}{I} \sum_{i=1}^{I} \tau_{1}^{i}-\tau_{2}^{i}
$$

will dictate the label of $s^{\prime}$ and $s^{\prime \prime}$. If $\tilde{\tau}$ is positive then $s^{\prime}$ will be labeled as $s 1$ and $s^{\prime \prime}$ as s2. The opposite will happen if $\tilde{\tau}$ is negative.

Because the detection is made in the frequency domain and the resolution is given by the onset detection function moving window step size, from equation (2) we will end up having a time domain resolution of $N / 8=F s / 12 * 1 / 8=F s / 96$ samples. In order to further improve the results, for every detected s1 and s2 we added supplementary local peak detection, within the interval $\pm F s / 48$ samples, centered on the detected s position.

\section{EXPERIMENTAL RESULTS}

The method presented here has been tested in terms of sensitivity, specificity and detection accuracy expressed in milliseconds and also as a percent of the beat period. 18 heart sound recordings were analyzed, containing normal $(\mathrm{N})$ and pathological sounds (the pathologies are: Aortic Stenosis AS, Aortic Insufficiency - AI, Mitral Regurgitation - MR, Mitral Valve Prolapse - MVP, Pulmonic Stenosis - PS, Tricuspid Stenosis - TS).

The mean value

$$
\bar{\varepsilon}=\frac{1}{n} \sum_{i=1}^{n} \varepsilon_{i}
$$

and also the standard deviation

$$
s=\sqrt{\frac{1}{n-1} \sum_{i=1}^{n}\left(\varepsilon_{i}-\bar{\varepsilon}\right)^{2}}
$$

were calculated for each recording.

To determine the optimal parameter set $(\delta ; \sigma)$ we repeatedly analyzed all the recordings and calculated the average absolute error. The parameter set that produced the smallest detection errors was $(\delta=0.95 ; \sigma=T / 4.3)$ and it was used for all the simulations that are presented from this point forward.

Table 2 shows the obtained average detection errors expressed in $\mathrm{ms}$ and also in percent of the beat period. Positive values indicate early detection and negative values indicate late detection. Overall the absolute value mean error was of $14.2 \mathrm{~ms}$ for $\mathrm{s} 1$ and $34.4 \mathrm{~ms}$ for $\mathrm{s} 2(1.74 \%$ of the beat period for s1 and $2.37 \%$ for s2).

It can be observed that because the method is based on onset, the algorithm has a tendency of detecting s1 and s2 earlier than they actually occur thus the majority of the values in milliseconds have positive values. In terms of sensitivity and specificity the results obtained were very good, and even more important with no false negative detections across all recordings (Table 1). The false positive detections appeared in all situations at the beginning of the recording and are attributed to the fact that the detection method needs a short

\begin{tabular}{|c|c|c|c|c|c|c|c|c|c|}
\hline 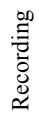 & 营 & 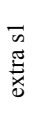 & 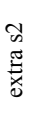 & $\begin{array}{l}\vec{n} \\
\tilde{d}^{0} \\
. \\
. \\
\Xi\end{array}$ & 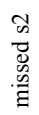 & 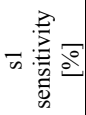 & 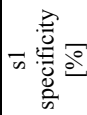 & 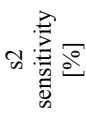 & 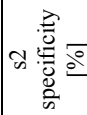 \\
\hline 1 & 25 & 0 & 0 & 0 & 0 & 100 & 100 & 100 & 100 \\
\hline 2 & 6 & 0 & 1 & 0 & 0 & 100 & 100 & 100 & 85.7 \\
\hline 3 & 22 & 0 & 1 & 0 & 0 & 100 & 100 & 100 & 95.7 \\
\hline 4 & 25 & 0 & 0 & 0 & 0 & 100 & 100 & 100 & 100 \\
\hline 5 & 23 & 1 & 1 & 0 & 0 & 100 & 95.8 & 100 & 95.8 \\
\hline 6 & 25 & 0 & 0 & 0 & 0 & 100 & 100 & 100 & 100 \\
\hline 7 & 17 & 0 & 1 & 0 & 0 & 100 & 100 & 100 & 94.4 \\
\hline 8 & 34 & 0 & 1 & 0 & 0 & 100 & 100 & 100 & 97.1 \\
\hline 9 & 37 & 1 & 1 & 0 & 0 & 100 & 97.4 & 100 & 97.4 \\
\hline 10 & 8 & 0 & 1 & 0 & 0 & 100 & 100 & 100 & 88.9 \\
\hline 11 & 20 & 0 & 1 & 0 & 0 & 100 & 100 & 100 & 95.2 \\
\hline 12 & 24 & 0 & 1 & 0 & 0 & 100 & 100 & 100 & 96.0 \\
\hline 13 & 10 & 0 & 0 & 0 & 0 & 100 & 100 & 100 & 100 \\
\hline 14 & 19 & 0 & 1 & 0 & 0 & 100 & 100 & 100 & 95.0 \\
\hline 15 & 24 & 1 & 1 & 0 & 0 & 100 & 96.0 & 100 & 96.0 \\
\hline 16 & 19 & 1 & 1 & 0 & 0 & 100 & 95.0 & 100 & 95.0 \\
\hline 17 & 13 & 0 & 1 & 0 & 0 & 100 & 100 & 100 & 92.9 \\
\hline 18 & 17 & 0 & 1 & 0 & 0 & 100 & 100 & 100 & 94.4 \\
\hline
\end{tabular}
period of time to "latch on" to the beats.

TABLE 1 . Sensitivity and specificity the results for each recording

By comparing these results with those obtained in literature, it can be seen that although high accuracy (less than $10 \mathrm{~ms}$ ) is not obtained overall, it is compensated by the very good results in terms of correct detection: the selectivity and specificity are close to $100 \%$ or even $100 \%$ if the above mentioned situations are ignored. By comparison, in [5] the reported selectivity and specificity were around $91 \%$ and the reported average detection accuracy was of approximately 4.2 $\mathrm{ms}$.

The greatest advantage of our method is that because it is based on periodicity, transient noises that are generated either by physiological or environmental factors can have little influence, if any, on the detection results. To show this we ran the algorithm on the same recordings contaminated with noise. We used additive pink noise, with normalized amplitude equal to $20 \%$ of the recording amplitude. The results, presented in table 3 clearly show that as we expected, there is little difference between the two situations and the detection still performs good. 
TABLE 2. Average detection errors expressed in ms and also in percent of the beat period for every recording; positive values indicate early detection and negative values indicate late detection

\begin{tabular}{|c|c|c|c|c|c|c|c|c|c|c|c|}
\hline 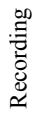 & 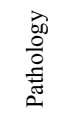 & $\sum_{i=1}$ & $\begin{array}{l}\text { Total } \\
\text { number of } \\
\text { beats in } \\
\text { recording }\end{array}$ & $\begin{array}{l}\text { Mean s1 } \\
\text { error }[\mathrm{ms}]\end{array}$ & $\begin{array}{c}\text { Standard } \\
\text { deviation of s1 } \\
\text { error }[\mathrm{ms}]\end{array}$ & $\begin{array}{c}\text { Mean s2 } \\
\text { error } \\
{[\mathrm{ms}]}\end{array}$ & $\begin{array}{c}\text { Standard } \\
\text { deviation of s2 } \\
\text { error }[\mathrm{ms}]\end{array}$ & $\begin{array}{l}\text { Mean s1 } \\
\text { error as \% } \\
\text { of period }\end{array}$ & $\begin{array}{c}\text { Standard } \\
\text { deviation of } 11 \\
\text { error as } \% \text { of } \\
\text { period }\end{array}$ & $\begin{array}{c}\text { Mean s } 2 \\
\text { error as } \% \text { of } \\
\text { period }\end{array}$ & $\begin{array}{l}\text { Standard deviation of } \mathrm{s} 1 \\
\text { error as } \% \text { of period }\end{array}$ \\
\hline 1 & AI & 65 & 25 & 816 & 2033 & -004 & 531 & 088 & 220 & 000 & 058 \\
\hline 2 & $\mathrm{AI}$ & 59 & 6 & -100 & 000 & -100 & 000 & -010 & 000 & -010 & 000 \\
\hline 3 & AS & 67 & 22 & -909 & 2392 & 336 & 2386 & $\begin{array}{ll}-102 \\
\end{array}$ & 267 & 038 & 266 \\
\hline 4 & AS & 75 & 25 & 596 & 3458 & 4896 & 9092 & 075 & 432 & 612 & 1137 \\
\hline 5 & AS & 80 & 23 & $\begin{array}{lll}1 & 04 \\
\end{array}$ & 047 & 144.74 & 5489 & -014 & 006 & 1930 & 732 \\
\hline 6 & AS & 79 & 25 & -520 & 344 & 13760 & 1718 & -068 & 045 & 1812 & 226 \\
\hline 7 & $\mathrm{~N}$ & 65 & 17 & 1529 & 2355 & 571 & 2837 & 166 & 255 & 062 & 307 \\
\hline 8 & $\mathrm{~N}$ & 103 & 34 & 1041 & 2241 & 144 & 1967 & 179 & 385 & 025 & 338 \\
\hline 9 & $\mathrm{~N}$ & 116 & 37 & 189 & 909 & -0.05 & 619 & 037 & 176 & $\begin{array}{llll}-0 & 01 \\
\end{array}$ & 120 \\
\hline 10 & $\mathrm{~N}$ & 66 & 8 & 425 & 1759 & -388 & 642 & 047 & 193 & -043 & 071 \\
\hline 11 & MR & 61 & 20 & 195 & 1014 & -090 & 045 & 020 & 103 & $\begin{array}{l}-009 \\
\end{array}$ & 005 \\
\hline 12 & MR & 73 & 24 & 1075 & 2703 & 2250 & 4771 & 131 & 329 & 274 & 580 \\
\hline 13 & MR & 54 & 10 & -090 & 057 & 1500 & 3413 & -008 & 005 & 135 & 307 \\
\hline 14 & MR & 76 & 19 & -5326 & 3131 & 6295 & 4200 & -675 & 397 & 797 & 532 \\
\hline 15 & MVP & 72 & 24 & -1788 & 6478 & 1917 & 3143 & -215 & 777 & 230 & 377 \\
\hline 16 & PS & 62 & 19 & -101.05 & 4922 & 984 & 3991 & -1044 & 509 & 102 & 412 \\
\hline 17 & PS & 78 & 13 & 415 & 659 & 8631 & 893 & 054 & 086 & 1122 & 116 \\
\hline 18 & TS & 70 & 17 & -0.82 & 113 & 088 & 683 & -010 & 013 & 010 & 080 \\
\hline
\end{tabular}

TABLE 3. Detection errors for each recording in the original and noiseaffected cases; original and noise affected results are placed side by side for a better comparison;

\begin{tabular}{|c|c|c|c|c|c|c|c|c|}
\hline Rec & $\begin{array}{c}\text { Mean } \\
\text { s1 error } \\
{[\mathbf{m s}]}\end{array}$ & $\begin{array}{c}\text { New } \\
\text { values } \\
{[\mathbf{m s}]}\end{array}$ & $\begin{array}{c}\text { Standard } \\
\text { deviation } \\
\text { of s1 error } \\
{[\mathrm{ms}]}\end{array}$ & $\begin{array}{c}\text { New } \\
\text { values } \\
{[\mathrm{ms}]}\end{array}$ & $\begin{array}{c}\text { Mean s2 } \\
\text { error } \\
{[\mathbf{m s}]}\end{array}$ & $\begin{array}{c}\text { New } \\
\text { values } \\
{[\mathbf{m s}]}\end{array}$ & $\begin{array}{c}\text { Standard } \\
\text { deviation } \\
\text { of s2 error } \\
{[\mathrm{ms}]}\end{array}$ & $\begin{array}{c}\text { New } \\
\text { values } \\
{[\mathrm{ms}]}\end{array}$ \\
\hline 1 & $\mathbf{8 . 1 6}$ & $\mathbf{7 . 6 4}$ & 2033 & 1573 & $\mathbf{- 0 . 0 4}$ & $\mathbf{0 . 3 6}$ & 531 & 585 \\
\hline 2 & $\mathbf{- 1 . 0 0}$ & $\mathbf{2 . 8 3}$ & 000 & 601 & $\mathbf{- 1 . 0 0}$ & $\mathbf{0 . 3 3}$ & 000 & 327 \\
\hline 3 & $\mathbf{- 9 . 0 9}$ & $\mathbf{1 7 . 8 2}$ & 2392 & 3838 & $\mathbf{3 . 3 6}$ & $\mathbf{1 5 . 7 7}$ & 2386 & 3080 \\
\hline 4 & $\mathbf{5 . 9 6}$ & $\mathbf{6 0 . 3 6}$ & 3458 & 14367 & $\mathbf{4 8 9 6}$ & $\mathbf{5 2 . 2 4}$ & 9092 & 9090 \\
\hline 5 & $\mathbf{- 1 . 0 4}$ & $\mathbf{- 1 . 0 4}$ & 047 & 056 & $\mathbf{1 4 4 . 7 4}$ & $\mathbf{1 4 0 . 7 8}$ & 5489 & 5479 \\
\hline 6 & $\mathbf{- 5 . 2 0}$ & $\mathbf{- 5 . 4 8}$ & 344 & 400 & $\mathbf{1 3 7 . 6 0}$ & $\mathbf{1 2 2} \mathbf{8 4}$ & 1718 & 1664 \\
\hline 7 & $\mathbf{1 5 . 2 9}$ & $\mathbf{2 5 . 5 3}$ & 2355 & 3692 & $\mathbf{5 . 7 1}$ & $\mathbf{1 4 . 5 3}$ & 2837 & 3347 \\
\hline 8 & $\mathbf{1 0 . 4 1}$ & $\mathbf{1 5 . 5 6}$ & 2241 & 2716 & $\mathbf{1 . 4 4}$ & $\mathbf{7 . 0 9}$ & 1967 & 1678 \\
\hline 9 & $\mathbf{1 . 8 9}$ & $\mathbf{1 1 . 2 4}$ & 909 & 2662 & $\mathbf{- 0 . 0 5}$ & $\mathbf{2 . 6 2}$ & 619 & 573 \\
\hline 10 & $\mathbf{4 . 2 5}$ & $\mathbf{4 . 7 5}$ & 1759 & 1785 & $\mathbf{- 3 . 8 8}$ & $\mathbf{- 4 . 0 0}$ & 642 & 630 \\
\hline 11 & $\mathbf{1 . 9 5}$ & $\mathbf{1 2 . 9 0}$ & 1014 & 1898 & $\mathbf{- 0 . 9 0}$ & $\mathbf{- 0 . 9 0}$ & 045 & 045 \\
\hline 12 & $\mathbf{1 0 . 7 5}$ & $\mathbf{3 6 . 1 3}$ & 2703 & 5592 & $\mathbf{2 2 5 0}$ & $\mathbf{3 6 . 9 6}$ & 4771 & 5149 \\
\hline 13 & $\mathbf{- 0 . 9 0}$ & $\mathbf{- 5 . 1 0}$ & 057 & 1403 & $\mathbf{1 5 . 0 0}$ & $\mathbf{6 . 8 0}$ & 3413 & 3230 \\
\hline 14 & $\mathbf{- 5 3 . 2 6}$ & $\mathbf{- 5 1 8 4}$ & 3131 & 3195 & $\mathbf{6 2} \mathbf{9 5}$ & $\mathbf{3 3 . 9 5}$ & 4200 & 3727 \\
\hline 15 & $\mathbf{- 1 7 8 8}$ & $\mathbf{- 1 8 5 4}$ & 6478 & 6373 & $\mathbf{1 9 1 7}$ & $\mathbf{1 9 . 3 3}$ & 3143 & 2791 \\
\hline 16 & $\mathbf{- 1 0 1 . 1}$ & $\mathbf{- 1 1 1 . 2}$ & 4922 & 4654 & $\mathbf{9 . 8 4}$ & $\mathbf{- 1 5 4 . 4}$ & 3991 & 10542 \\
\hline 17 & $\mathbf{4 . 1 5}$ & $\mathbf{1 2 . 2 3}$ & 659 & 1796 & $\mathbf{8 6 3 1}$ & $\mathbf{8 8 . 1 5}$ & 893 & 369 \\
\hline 18 & $\mathbf{- 0 . 8 2}$ & $\mathbf{- 1 . 7 1}$ & 113 & 285 & $\mathbf{0 . 8 8}$ & $\mathbf{- 0 . 2 4}$ & 683 & 1305 \\
\hline
\end{tabular}

In some cases, the detection results are identical, like in the case of $\mathrm{s} 1$ for recording 5 , where the s1 and s2 have high amplitude peaks. The $D F$ samples that are marked as s1 coincide in the two situations (no noise vs. noise), and because they exhibit high amplitudes the local peak detection that follows the beat tracking will return the same position. In terms of specificity and sensitivity the results remain identical with the original simulations.

\section{CONCLUSIONS}

We have proposed a robust method for heart sound segmentation and $\mathrm{s} 1 / \mathrm{s} 2$ detection, without ECG reference, based on a modified beat tracking algorithm. The particular advantages of using this method are that very good results are obtained in terms of sensitivity and specificity, and the presence of artifacts, noise, or murmurs only marginally affect the performance of the detector.
In terms of computational complexity, we ran the simulations on a $2 \mathrm{GHz}$ dual core AMD based machine with 2GB of RAM, using the MatlabR2010b environment and, on average, the analysis of one second of heart sound took 29 milliseconds. This is an important result because due to the small time needed for analysis a real-time version of the detector can be easily implemented.

\section{REFERENCES}

[1] M. E. P. Davies and M. D. Plumbley, "Context-Dependent Beat Tracking of Musical Audio", IEEE Trans. Audio, Speech, Lang. Process., Vol. 15, No. 3, March 2007, pp. 1009-1020;

[2] A. M. Stark, M. E. P. Davies and M. D. Plumbley, "Real-Time BeatSynchronous Analysis of Musical Audio", Proc. of the $12^{\text {th }}$ Int. Conference on Digital Audio Effects (DAFx-09), Como, Italy, September 1-4, 2009;

[3] J. P. Bello, L. Daudet, S. Abdallah, C. Duxbury, M. Davies, and M. B. Sandler, "A Tutorial on Onset Detection in Music Signals" IEEE Trans. Speech Audio Process., vol. 13, no. 5, pt. 2, pp. 1035-1047, Sep. 2005.

[4] H. Liang, S. Lukknen and I. Hartimo, "Heart Sound Segmentation Algorithm Based on Heart Sound Envelogram", Proc. of IEEE Computers in Cardiology, 1997, pp. 105-108;

[5] D. Kumar, P. Carvalho, M. Antunes, R. P. Paiva, J. Henriques, "An Adaptive Approach to Abnormal Heart Sound Segmentation", Proc. of International Conference on Acoustics, Speech, and Signal Processing (ICASSP), May 2011, Vol 2;

[6] D. Kumar, P. Carvalho, M. Antunes, P. Gil, J. Henriques, L. Eugenio "A New Algorithm For Detection Of S1 And S2 Heart Sounds", Proc. of International Conference on Acoustics, Speech, and Signal Processing (ICASSP), May 2006, pp. 661-664;

[7] P. Wang, Y. Kim, L. H. Ling and C. B. Soh, "First Heart Sound Detection for Phonocardiogram Segmentation", Engineering in Medicine and Biology 27th Annual Conference, Shanghai, China, September 2005, pp. 5519 5522;

[8] M.B. Malarvili, I. Kamarulafizam, S. Hussain, D. Helmi, "Heart Sound Segmentation Algorithm Based on Instantaneous Energy of Electrocardiogram", Proc. of Computers in Cardiology, Thessaloniki, Greece, 21-24 Sept. 2003, pp. 327-330; 